\title{
Myiasis in Children: Fly Larval Infestation
}

\author{
Meena $S^{1}$, Meena $D^{2}$
}

\begin{abstract}
Human myiasis is a parasitic infestation by fly larvae in poor hygienic or immunocompromised children's. We present here two case of fly larval infestation including one scalp myiasis in six year old male street child and other one vulvar myiasis in 18 year old unmarried girl suffering from insulin dependent diabetes mellitus and psychiatric illness.
\end{abstract}

Key words: Cutaneous myiasis, Vulvar myiasis, Poor hygiene.

\section{Introduction}

$\mathrm{M}$ yiasis is a parasitic infestation caused by the larvae of several species. Risk factors for human myiasis are poor hygiene, low education level and immunocompromised individuals ${ }^{1}$. Human myiasis can be classified based on clinical condition causing cutaneous myiasis, ocular myiasis, urogenital myiasis and intestinal myiasis ${ }^{2}$. We present here two case of fly larval infestation including one scalp myiasis in six year old male street child and other vulvar myiasis in 18 year old unmarried girl suffering from insulin- dependent diabetes mellitus and psychiatric illness.

\section{Case 1}

A six year street brought by a social worker to the paediatric emergency for scalp wound and complained about the presence of blood stained pus discharge. He was found with numerous live, larval forms in scalp wound. The symptoms were present for more than four weeks. He had loss of appetite, showed signs of fatigue and there were significant reduction in weight over one month. Initially about 10 maggots were removed using the non-toothed forceps, and the wound was cleaned with betadine. He was then treated with chloroform and turpentine oil in the ratio 1:4 which was followed by manual removal of the maggots $(n=20)$ and was made maggot-free in 2-3 days. He was put on cefuroxime empirically from the 1st day. He was also given a tetanus toxoid injection on admission. No complications were seen and was advised to take necessary precautions and prevent possible re-exposure along with prescription for nutritional and vitamin supplements.
'Dr. Shyam Sundar Meena, MBBS, MD, Consultant Paediatrician, Department of Paediatric Medicine, Lady Hardinge Medical College, New Delhi, India, ${ }^{2}$ Dr. Deepika Meena, MBBS, MS, Sr. Resident. Department of Obstetrics and Gynaecology, Maulana Azad Medical College, New Delhi, India.

\section{Address for correspondence:}

Dr. Shyam S Meena

E-mail: dr.shyamsnmc2000@gmail.com

\section{How to cite}

Meena SS, Meena D. Myiasis in Children: Fly Larval Infestation. J Nepal Paediatr Soc 2014;34(3):244246.

doi: http://dx.doi.org/10.3126/jnps.v34i3.10768

This work is licensed under a Creative Commons Attribution 3.0 License.

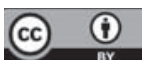

\section{Case 2}

An 18-year old unmarried girl was admitted to department of Obstetrics and Gynaecology with a history of vaginal discharge and vulval irritation for two week. She had also noted some worms around the vulva. Her hygiene was poor and as wearing dirty clothes. The patient was a known case of insulin dependent diabetes mellitus (IDDM) but under improper medication. She was also on antidepressant drugs. She was living with her relatives because her mother had died when she was a small child. There was a history of normal regular 


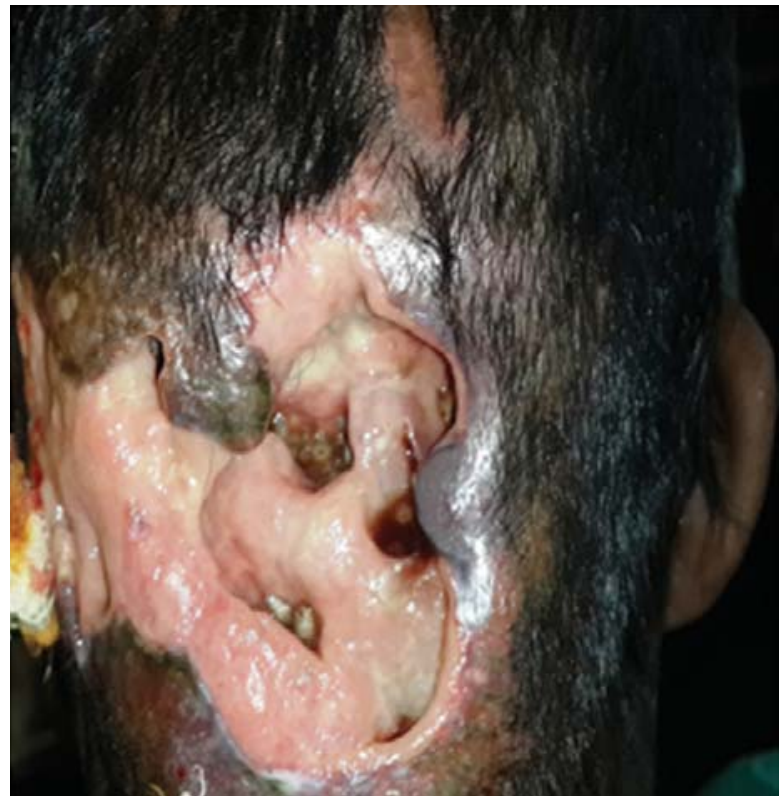

Fig 1: Showing myiasis of the scalp

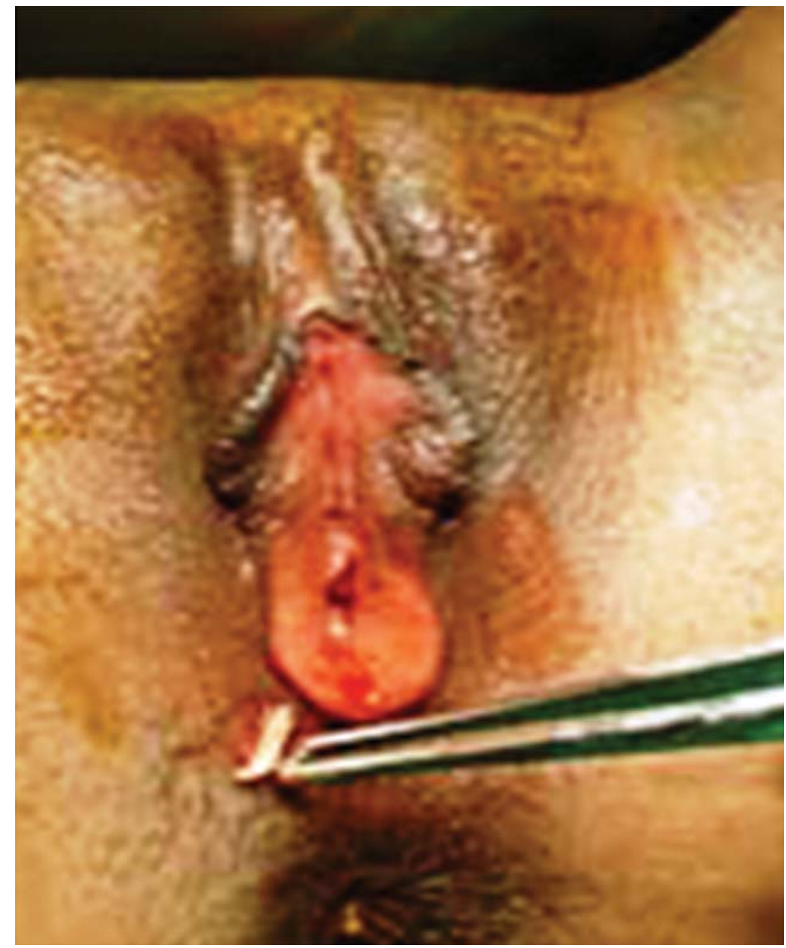

Fig 2: Showing myiasis of the Vulva

menstrual cycle prior to the onset of this discharge from genitalia. Patient did not use sanitary napkins during the menstrual periods; instead, she used dirty ragged clothes.

On examination, there was oedema and inflammation of the labia minora. Few maggots were found on the perineum. An examination under local anaesthesia revealed an ulcer on the inner surface of the left labium minora involving the hymen ring as well. The ulcer was infested by a large number of maggots. No maggots were found in the vagina or rectum. Initially about 31 magots were removed with help of non-toothed forceps and clean the wound with betadine. Antibiotics (Ceftriaxone, metronidalzole and doxycylcline) were started empirically. She was also received tetanus toxoid injection on admission. By the second day, turpentine oil was applied on the ulcer. By the 4th day, with 3 days of treatment with turpentine oil, the maggots had completely disappeared and the ulcer was healing. Patient was followed up for four weeks and was found to be completely cured. She was given advice on personnel hygiene to avoid reinfestation.

In both cases larvae were preserved in laboratory for development of adult fly. After development in to adult fly it was confirmed as belonging to the house fly.

\section{Discussion}

Myiasis is defined as a disease caused by the infiltration of body tissues by larvae of several fly species of veterinary and medical interest. It occurs predominantly in rural areas and is associated with poor hygiene and low educational level ${ }^{1,2}$. It is also common in children ${ }^{1}$. Human myiasis can be classified based on clinical condition it causes like cutaneous myiasis, ocular myiasis, urogenital myiasis and intestinal myiasis ${ }^{2}$. Maggots can enter through intact skin or through a wound. Very few cases have been retrieved from literature on the occurrence of scalp and vulvar myiasis in children throughout the world. Probably due to under reporting many clinicians and pediatricians still have inadequate knowledge about clinical implications of human myiasis. Vulvar myiasis constitutes only $0.7 \%$ of human infestation ${ }^{4}$. We consider our both patients as a case of myiasis as the maggots have invaded the scalp and vulvar tissue. The most common species that infest wounds in USA is found to be Phaenicia sericata ${ }^{4}$. The fly species that infest healthy tissue are called Biontophages and responsible for the cutaneous forms of this condition (furunculoid myiasis) ${ }^{1}$. Complications include secondary infections and tetanus. Treatment is removal of larvae and thorough cleaning of the affected area. Applying turpentine oil makes the respiration impossible for the larvae and is one modality of treatment ${ }^{1}$. A small surgical procedure may be required to remove larvae and after removal the area should be cleaned and covered with an antibiotic ointment ${ }^{4}$. Treatment of secondary infection and administering tetanus toxoid is mandatory.

As poor hygiene is known to be associated with vulvar myiasis ${ }^{5}$ washing and keeping the genital area 
clean may prevent to a great extent the occurrence of this condition. It is important to exclude sexually transmitted diseases as a high proportion of cases had sexually transmitted diseases including HIV ${ }^{1,2}$. Sherman recommends maggots to be submitted for species identification ${ }^{4}$. Species identification is important for determining pathogenesis, its potential invasiveness, in determining the exact timing and circumstances of infestation.

Turpentine oil has been advocated in many cases of cutaneous myiasis worldwide ${ }^{6}$ but none have mentioned its use in vulvar myiasis. In both cases, the use of turpentine oil produced excellent results.

\section{Conclusion}

We re-emphasize the role of human myiasis and the associated morbidity it can cause, as evidenced by increasing reports worldwide. Children are predisposed more to infestation with fly larvae due to their playing habits and lack of knowledge of hygiene, especially in rural areas. Paediatricians should be aware of myiasis causing larvae and advise from the microbiologists for laboratory identification and also initiate appropriate supportive treatment wherever necessary to minimize the morbidity.

\section{References}

1. Passos MRL, Varella RQ, Tavares RR, Barreto NA, Santos CCC, Pinheiro VMS, et al. Vulvar myiasis during pregnancy. 2002;10(3): 153-58.

2. Zumpt F 1965. Myiasis in man and animals in the Old World. Butterworths, London, United Kingdom.

3. Predy G, Angus M, Honish L, Burnett CE, Stagg A. Myiasis in an urban setting: A case report. Can J Infect Dis 2004;15(1):51-2.

4. Sherman RA. Wound myiasis in urban and suburban United States. Arch Int Med 2000;60:2004-14.

5. Nunzi E, Rongioletti F, Rebora A. Removal of Dermatobia hominis larvae. Arch Dermatol 1986;122(2):140.

6. Yazar S, Ozcan H, Dincer S, Sahin I. Vulvar myiasis. Yonsei Med J 2002;43(4):553-55.

7. Sharma J, Mamatha GP and Acharya R. Primary oral myiasis: A case report. Med Oral Patol Oral Cir Bucal 2008;13(11):E714-6. 